\title{
Sinogram bow-tie filtering in FBP PET reconstruction
}

\author{
M. Abella and J. J. Vaquero \\ Unidad de Medicina y Cirugía Experimental, Hospital General Universitario Gregorio Marañón, \\ 28007 Madrid, Spain
}

M. L. Soto-Montenegro

Unidad de Medicina y Cirugía Experimental, Hospital General Universitario Gregorio Marañón, 28007 Madrid, Spain and Centro de Investigación en Red de Salud Mental (CIBERSAM, CIBER CB07/09/0031), 28007 Madrid, Spain

E. Lage

Unidad de Medicina y Cirugía Experimental, Hospital General Universitario Gregorio Marañón, 28007 Madrid, Spain

M. Desco b)

Unidad de Medicina y Cirugía Experimental, Hospital General Universitario Gregorio Marañón, 28007 Madrid, Spain and Centro de Investigación en Red de Salud Mental (CIBERSAM, CIBER CB07/09/0031), 28007 Madrid, Spain

Low-pass filtering of sinograms in the radial direction is the most common practice to limit noise amplification in filtered back projection (FBP) reconstruction of positron emission tomography studies. Other filtering strategies have been proposed to prevent the loss in resolution due to low-pass radial filters, although results have been diverse. Using the well-known properties of the Fourier transform of a sinogram, the authors defined a binary mask that matches the expected shape of the support region in the Fourier domain of the sinogram ("bow tie"). This mask was smoothed by a convolution with a ten-point Gaussian kernel which not only avoids ringing but also introduces a pre-emphasis at low frequencies. A new filtering scheme for FBP is proposed, comprising this smoothed bow-tie filter combined with a standard radial filter and an axial filter. The authors compared the performance of the bow-tie filtering scheme with that of other previously reported methods: Standard radial filtering, angular filtering, and stackgram-domain filtering. All the quantitative data in the comparisons refer to a baseline reconstruction using a ramp filter only. When using the smallest size of the Gaussian kernel in the stackgram domain, the authors achieved a noise reduction of $33 \%$ at the cost of degrading radial and tangential resolutions $(14.5 \%$ and $16 \%$, respectively, for cubic interpolation). To reduce the noise by $30 \%$, the angular filter produced a larger degradation of contrast (3\%) and tangential resolution $(46 \%$ at $10 \mathrm{~mm}$ from the center of the field of view) and showed noticeable artifacts in the form of circular blurring dependent on the distance to the center of the field of view. For a similar noise reduction (33\%), the proposed bow-tie filtering scheme yielded optimum results in resolution (gain in radial resolution of 10\%) and contrast ( $1 \%$ increase) when compared with any of the other filters alone. Experiments with rodent images showed noticeable image quality enhancement when using the proposed bow-tie filtering scheme.

Key words: PET reconstruction, filtered backprojection, FBP, bow-tie, sinogram filtering, noise reduction

\section{INTRODUCTION}

One of the main shortcomings of data acquired using positron emission tomography (PET) systems is the low number of detected events (usually referred to as "low statistics"), which results in noisy reconstructions. In the classic reconstruction algorithm, filtered back projection (FBP), noise reduction is achieved by performing low-pass filtering in the radial direction of the sinogram, at the cost of degrading resolution. Several works have studied the effect of alternative strategies for noise reduction. Daube-Witherspoon and Carson $^{1,2}$ analyzed the effect of angular and axial filtering on noise and resolution. One of their contributions was to characterize the nonuniform blurring produced by the angular filter.

Chatziioannou and Dahlbom ${ }^{3}$ studied the effect of radial and axial filtering in transmission and emission images in the context of attenuation correction for PET. Happonen and Alenius proposed a new sinogram decomposition by defining a new domain, the so-called stackgram domain. ${ }^{4,5}$ In this domain, 2D projection data are represented as a volume, where each slice is the back projection of one row of the sinogram. This space enables easy filtering of each point in the image following its sinusoidal trajectory in the sinogram, 

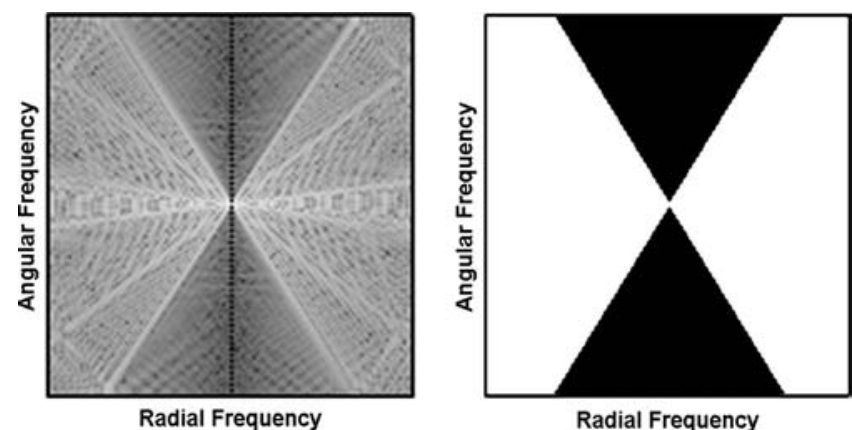

FIG. 1. 2D Fourier transform of a sinogram (left) and basic bow-tie mask in the FT domain for $\xi=1$ (right).

which turns into a straight line in the stackgram domain (vertical direction across the slices). This filtering scheme was claimed to prevent blurring artifacts both in the radial and angular dimensions.

On the other hand, Rattey and Lindgren ${ }^{6}$ studied the 2D Fourier transform of the sinogram to find its optimum sampling and observed that the support region of the frequency content of any sinogram formed what they called a bow-tie shape (Fig. 1, left panel). The bow-tie shape described by Rattey and Lindgren implies that the support in the radial direction is limited by the highest frequency in the image, $w_{\max }$ (determined by the acquisition process), while the limit in the angular direction depends on the radius of the object, $R_{M}$, according to the expression $R_{M}\left|w_{\max }\right|+1$ (Fig. 1, left panel). A thorough discussion of these properties can be found in Edholm et al. ${ }^{7}$ The Fourier rebinning algorithm, a rebinning method widely used in PET studies to compact a $3 \mathrm{D}$ acquisition dataset into a stack of ordinary 2D data, ${ }^{8,9}$ makes use of this property through the so-called consistency condition for the 2D Radon transform. ${ }^{10}$ In this case, it is common not to take into account the actual radius of support (object dependent) but to consider the whole field of view (FOV). The bow-tie property has also been used to estimate missing projection data in PET (Ref. 11) and to compensate for spatially variant collimator blurring in single photon emission computed tomography. ${ }^{12}$ Also, Andía ${ }^{13}$ exploited this property to develop optimal nonstationary linear filters based on sinogram statistics.

The main objective of our work was to take advantage of the bow-tie property to develop a new filtering scheme. We compare the results of this filtering scheme with those provided by previous standard techniques (stationary 1D filtering of the sinogram in the radial and angular directions) and by filtering in the stackgram domain as proposed by Happonen and Alenius. ${ }^{4}$ We study resolution, contrast, and noise by giving the differences (as a percentage) with respect to a baseline reconstruction consisting of FBP using the ramp filter only. Results are also presented for rodent images.

\section{MATERIAL AND METHODS}

\section{II.A. Data acquisition and reconstruction}

Data used in this work were obtained using an rPET scanner (SUINSA Medical Systems, Madrid, Spain). ${ }^{14,15}$ This system has four detectors positioned as two orthogonal pairs. Each detector comprises an array of $30 \times 30$ crystals of mixed lutetium silicate $\left(1.5 \times 1.5 \times 12 \mathrm{~mm}^{3}\right)$ optically coupled to a Hamamatsu H8500 position sensitive photomultiplier. The system rotates $180^{\circ}$ covering a total angle of $194.4^{\circ}$. List data are rearranged into $180^{\circ}$ sinograms with 120 angular bins and 55 radial bins. The sinogram spacing is $0.8 \mathrm{~mm}$ and $1.5^{\circ}$ in the radial and angular directions, respectively.

Reconstruction was performed by means of a single slice rebinning algorithm, ${ }^{16}$ followed by a $2 \mathrm{D}-\mathrm{FBP}$ reconstruction algorithm. The voxel size of the resulting image was $0.8 \mathrm{~mm}$ isotropic. All the filtering schemes tested in this work were incorporated into this reconstruction algorithm.

\section{II.B. Bow-tie filtering scheme}

The bow-tie filtering scheme proposed was based on applying a mask in the Fourier domain of the sinogram data. A binary bow-tie-shaped mask, $M$, was defined by the following expression:

$$
M\left(w_{r}, w_{\theta}\right)=\operatorname{rect}\left(w_{r}, w_{\max }\right) \times \operatorname{rect}\left(w_{\theta},\left[\left|w_{r}\right| \cdot \xi+1\right]\right),
$$

where $w_{r}$ is the radial frequency, $w_{\theta}$ is the angular frequency, $w_{\max }$ is maximum frequency in the image, and $\operatorname{rect}\left(w_{r}, w_{\theta}\right)$ is defined by the following expression:

$$
\begin{gathered}
\operatorname{rect}(x, a) \begin{cases}1, & |x| \leqslant a \\
0, & \text { otherwise }\end{cases} \\
\left\{\begin{array}{c}
\omega_{r} \in[-1,1], \omega_{\theta} \in\left[-\frac{\pi}{2}, \frac{\pi}{2}\right] \\
\xi=\frac{R_{M}}{\text { FOV_radius }} \times \frac{\pi}{2} .
\end{array}\right.
\end{gathered}
$$

This binary mask (shown in Fig. 1, right) was smoothed by a convolution with a $15 \times 15$-pixel Gaussian kernel. This procedure not only avoids ringing but also introduces a preemphasis at low frequencies.

The complete filtering scheme proposed is a combination of this smoothed bow-tie filter with a standard radial filter and an additional axial filter, which provides further noise reduction and improved isotropy without degrading transaxial resolution.

We studied the effect of the proposed scheme and different intermediate implementations (the nonsmoothed binary mask, $M$, the smoothed bow-tie filter, and the smoothed bow-tie filter combined only with a radial filter) for $\xi$ values ranging from 0.20 to 1 in steps of 0.05 . The value 1 corresponds to $100 \%$ of the FOV.

\section{II.C. Radial, angular, stackgram, and axial filters}

The implementation of the radial, angular, and stackgramdomain filters used in the analysis was as follows. Radial filter: Butterworth kernels (12th-order) with 20 different cutoff frequencies (5\%-100\% of the Nyquist frequency in steps of 5\%). Angular filter: Spatially invariant Gaussian kernels with ten different full width at half maximum (FWHM) val- 


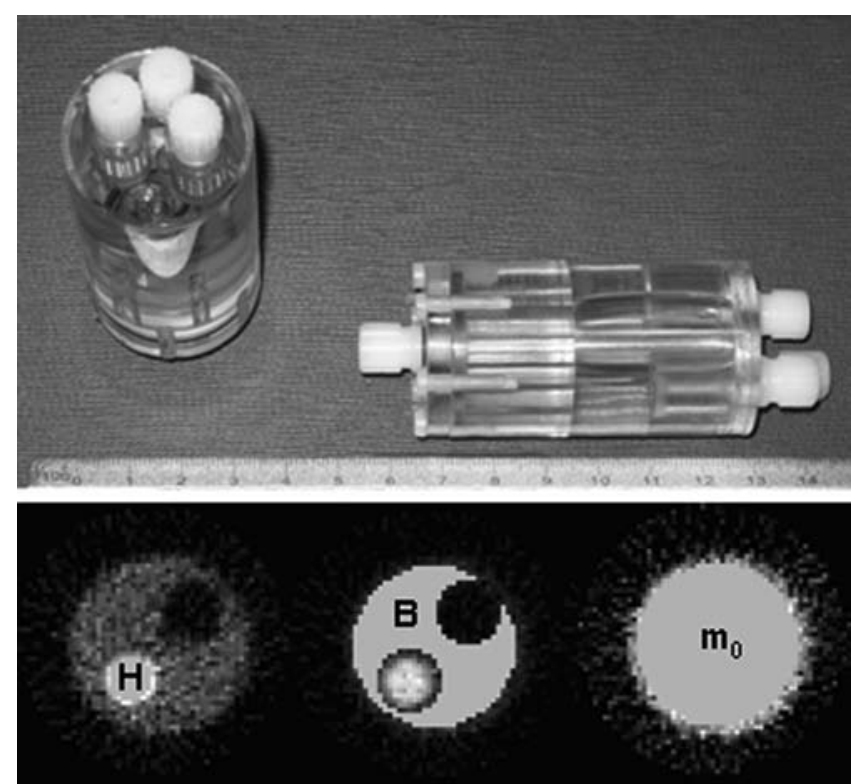

FIG. 2. Top: Image quality phantom used for contrast and noise measurement. Bottom left and center panels show one slice of the VOIs defined for contrast assessment ( $H$, hot; $B$, background). Bottom right panel shows a different part of the phantom where the VOI for noise measurement was drawn $\left(m_{0}\right)$.

ues $\left(1.7^{\circ}-18.7^{\circ}\right.$ in steps of $\left.1.7^{\circ}\right)$. Stackgram-domain filter: Gaussian kernels with ten different FWHM values (0.94$10.34 \mathrm{~mm}$ in steps of $0.94 \mathrm{~mm}$ ). These were all implemented with three interpolation methods: nearest neighbor, linear, and cubic. Axial filter: Gaussian kernels with FWHM values of 0.94 and $1.88 \mathrm{~mm}$.

The shapes of these filters and the range of their parameters were selected according to values proposed in literature and commonly used in nuclear medicine. Gaussian kernels have been used for angular and axial filtering in PET. ${ }^{1,2}$ From the several approaches proposed for radial filtering, the Butterworth low-pass kernel is among the most commonly used ones in FBP reconstruction. ${ }^{17}$

\section{II.D. Assessment of results}

To obtain quantitative measurements of contrast, we used the image quality phantom developed by NEMA (http:// www.nema.org/stds/nu4.cfm), shown in Fig. 2, top. The top part of the phantom is a $19.5 \mathrm{ml}$ cylinder containing two inner cylinders of $0.75 \mathrm{ml}$. One of the inner cylinders is empty and the other is filled with approximately four times more activity concentration than the background cylinder $[C(H)=30.85 \mu \mathrm{Ci} / \mathrm{ml}, C(B)=7.17 \mu \mathrm{Ci} / \mathrm{ml}$, and $C(H) / C(B)=4.30]$. The phantom was placed with its axial axis aligned with the axis of rotation of the scanner and imaged for $20 \mathrm{~min}$.

To evaluate the effect of the different filtering schemes on the contrast, we defined two volumes of interest (VOIs) corresponding to the high-activity region and background of the phantom: $H$ and $B$ in Fig. 2, bottom. $H$ is a 9 pixel $(7.2 \mathrm{~mm})$ diameter cylindrical region comprising six slices of the phantom; $B$ is a 33 pixel $(26.4 \mathrm{~mm})$ diameter cylinder comprising
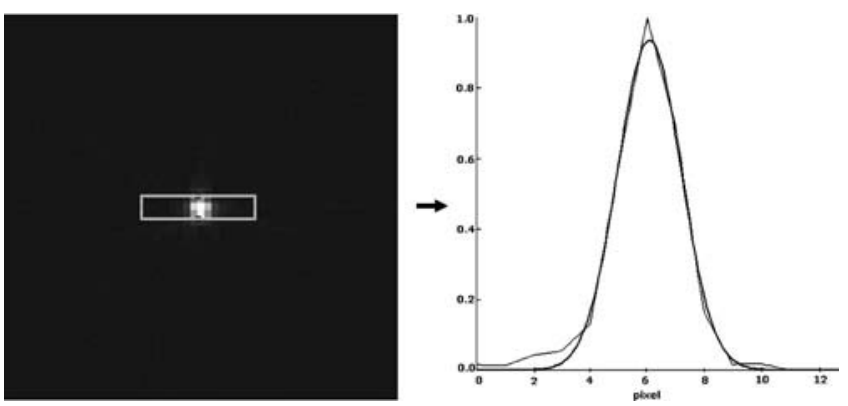

FIG. 3. One slice of the point source study. The profile was obtained from an ROI of $4 \times 13$ pixels and fitted to a Gaussian function (thick line).

six slices with two cylindrical holes of 13 pixel $(10.4 \mathrm{~mm})$ diameter inside. In order to assess image noise, we defined one more cylindrical VOI with a diameter of 33 pixels in 15 slices in the homogeneous part of the phantom $\left(m_{0}\right.$ in Fig. 2 , bottom).

Relative contrast $(\mathrm{RC})$ and noise $\left(N_{0}\right)$ were measured as

$$
\begin{aligned}
& \mathrm{RC}=\frac{\mu_{H}-\mu_{B}}{\left(\frac{\mu_{B}+\mu_{B}}{2}\right)}, \\
& N_{0}=\frac{\sigma_{m_{0}}}{\mu_{m_{0}}},
\end{aligned}
$$

where $\mu$ is the mean, $\sigma$ is the standard deviation, and $H, B$, and $m_{0}$ are the VOIs as depicted in Fig. 2, bottom.

To assess resolution, we used a point source that was $0.5 \mathrm{~mm}$ in diameter and $1 \mathrm{~mm}$ in length, placed at four different radial positions of the FOV: Offsets from the center of the FOV equal to $0,5,10$, and $15 \mathrm{~mm}$. The radial and tangential profiles of each point source were taken by drawing a region of interest (ROI) of $4 \times 13$ pixels containing the point. The profile was fitted to a Gaussian function by using a Powell optimization algorithm (Fig. 3). This process was repeated for three slices and FWHM values were averaged.

To further assess the performance of our procedure, we conducted three animal studies with fluorodeoxyglucose (FDG) on rodents: A 60 min FDG rat brain scan $(212 \mathrm{~g}$, $2.1 \mu \mathrm{Ci}$, and $30 \mathrm{~min}$ uptake), a $120 \mathrm{~min}$ FDG mouse brain scan ( $28 \mathrm{~g}, 563 \mu \mathrm{Ci}$, and $40 \mathrm{~min}$ uptake), and a $60 \mathrm{~min}$ FDG mouse heart scan $(27 \mathrm{~g}, 605 \mu \mathrm{Ci}$, and 40 min uptake). Quantitative data were also measured from the mouse heart study in terms of peak-to-valley ratio of a profile across the myocardium. We selected this study for quantification because the expected ideal profile is well known and the heart has also been used for similar comparisons elsewhere. ${ }^{18,17,19}$

All the results presented are expressed as a percentage with respect to a baseline reconstruction consisting of FBP with a ramp filter only.

\section{RESULTS}

Table I shows the filters used in the comparison. Only results for one instance of radial and axial filters in the proposed filtering scheme for a $\xi$ value matched to the size of 
TABLE I. Filters used in the comparison.

\begin{tabular}{|c|c|c|}
\hline Filtering scheme & Type of filter & Parameter \\
\hline Radial & Butterworth & $\begin{array}{l}\text { Cutoff frequencies: } 5 \%-100 \% \text { of the Nyquist } \\
\text { frequency in steps of } 5 \%\end{array}$ \\
\hline Angular & Gaussian & FWHM: $1.7^{\circ}-18.7^{\circ}$ in steps of $1.7^{\circ}$ \\
\hline Stackgram & Gaussian & FWHM: $0.94-10.34 \mathrm{~mm}$ in steps of $0.94 \mathrm{~mm}$ \\
\hline Binary bow-tie & Binary mask [M as defined in Eqs. (1) and (2)] & $\xi: 0.20-1$ in steps of 0.05 \\
\hline Smoothed bow-tie & $\begin{array}{l}\text { Binary bow-tie mask smoothed with a } \\
\qquad 15 \times 15 \text {-pixel Gaussian kernel }\end{array}$ & Bow-tie $\xi: 0.20-1$ in steps of 0.05 \\
\hline Smoothed bow-tie + radial & Smoothed bow-tie + radial filter, 12th-order & $\begin{array}{l}\text { Butterworth cutoff frequency }=50 \% \text { of the Nyquist } \\
\text { frequency. Bow-tie } \xi: 0.20-1 \text { in steps of } 0.05\end{array}$ \\
\hline Smoothed bow-tie + radial + axial & $\begin{array}{l}\text { Smoothed bow-tie }+ \text { Butterworth radial filter, } \\
\text { 12th-order }+ \text { Gaussian filter in the axial direction }\end{array}$ & $\begin{array}{l}\text { Butterworth cutoff frequency }=50 \% \text { of the Nyquist } \\
\text { frequency. Gaussian FWHM }=0.94 \mathrm{~mm} \text {. } \\
\text { Bow-tie } \xi: 0.20-1 \text { in steps of } 0.05\end{array}$ \\
\hline
\end{tabular}

the phantom are presented $(\xi=0.75$, as $75 \%$ of the FOV is filled). We also present the results obtained with intermediate realizations of our proposed scheme, i.e., binary bow-tie mask, after smoothing with the Gaussian kernel, and after applying the radial filter.

Figure 4 shows the results for the three interpolation schemes tested for the stackgram filter: Nearest neighbor (diamonds), linear interpolation (squares), and cubic (triangles). All of them produced different degrees of resolution degradation even when no filtering was applied: Increase in the root-mean-square FWHM of $16 \%$ for nearest-neighbor interpolation, $18 \%$ for linear interpolation, and $7 \%$ for cubic interpolation (" ," " $\mathbf{\square}$," and " $\boldsymbol{\Delta}$," respectively, in Fig. 4, right). At the same time, the noise reduction achieved without any filter applied was 14\% for nearest neighbor, 35\% for linear, and 19\% for cubic interpolation (" ," " $\mathbf{\square}$," and " $\mathbf{\Lambda}$," respectively, in Fig. 4, left), and contrast decreased in 3\% for the worst case, i.e., nearest interpolation (" $\bullet$ " in Fig. 4, left). For comparison with the rest of the filtering schemes, only results for cubic interpolation scheme are shown in the plots.

Figures 5 and 6 show the results for noise and contrast expressed as a percentage of change with respect to a base- line reconstruction (ramp filter only), and resolution (both tangential and radial). The following results are presented filter by filter, referencing Fig. 5 for contrast vs. noise and Fig. 6 for resolution. As the performance of any filter is usually a trade-off between noise reduction and resolution degradation, comparison is not obvious because the filter with the best performance will depend on which effect is sought. In order to be able to extract some conclusions from the comparison, the figures given in the following paragraphs show the effect of each filter for a realization that achieves a minimum noise reduction of $30 \%$, which corresponds with the noise reduction obtained with the proposed filter.

As compared with the baseline reconstruction, the radial filter (depicted as "+" in Figs. 5 and 6) reduces noise without a noticeable impact on contrast, for reasonable cutoff frequency values (over $20 \%$ of Nyquist frequency). For a reduction in noise of $32 \%$, we had a $14 \%$ loss of radial resolution (Fig. 6, right) and a 6\% loss of tangential resolution (Fig. 6, left).

For a similar reduction in noise (30\%), the angular filter (depicted as " $X$ " in Figs. 5 and 6) produced more contrast

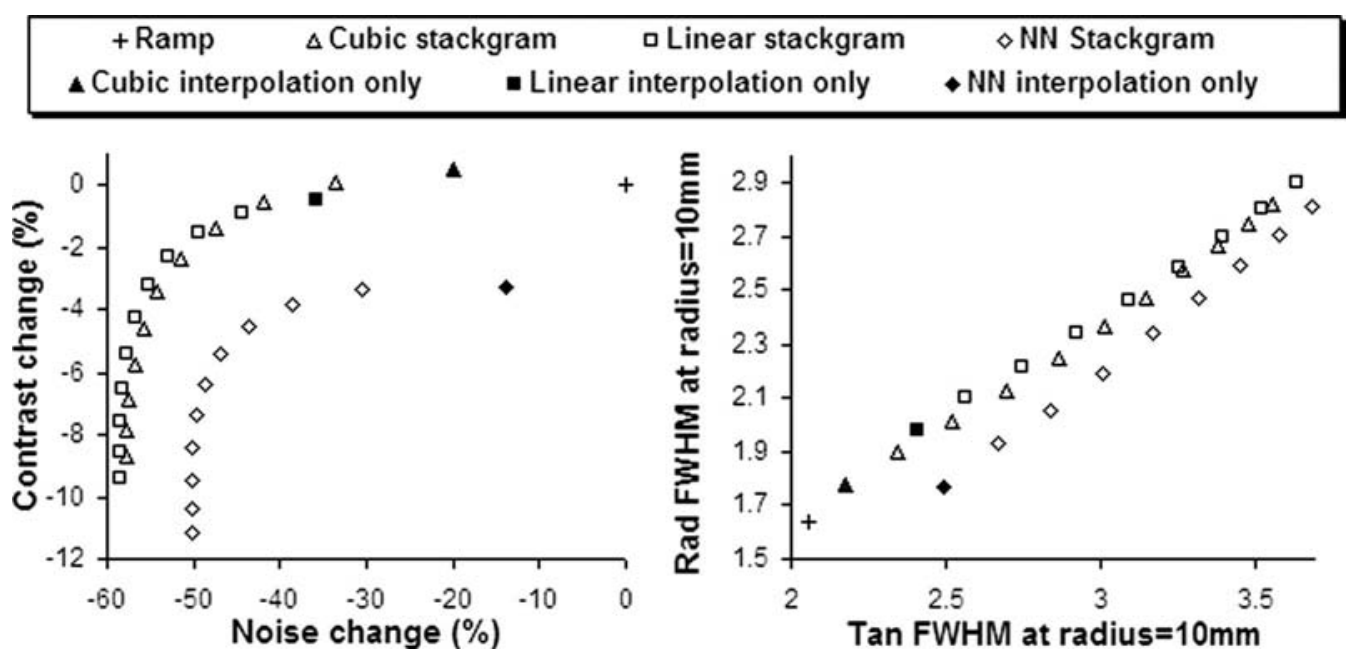

FIG. 4. Results of stackgram filtering for the three interpolation schemes tested. Filled symbols show the results of just transforming to the stackgram domain and back, with no filtering applied. 


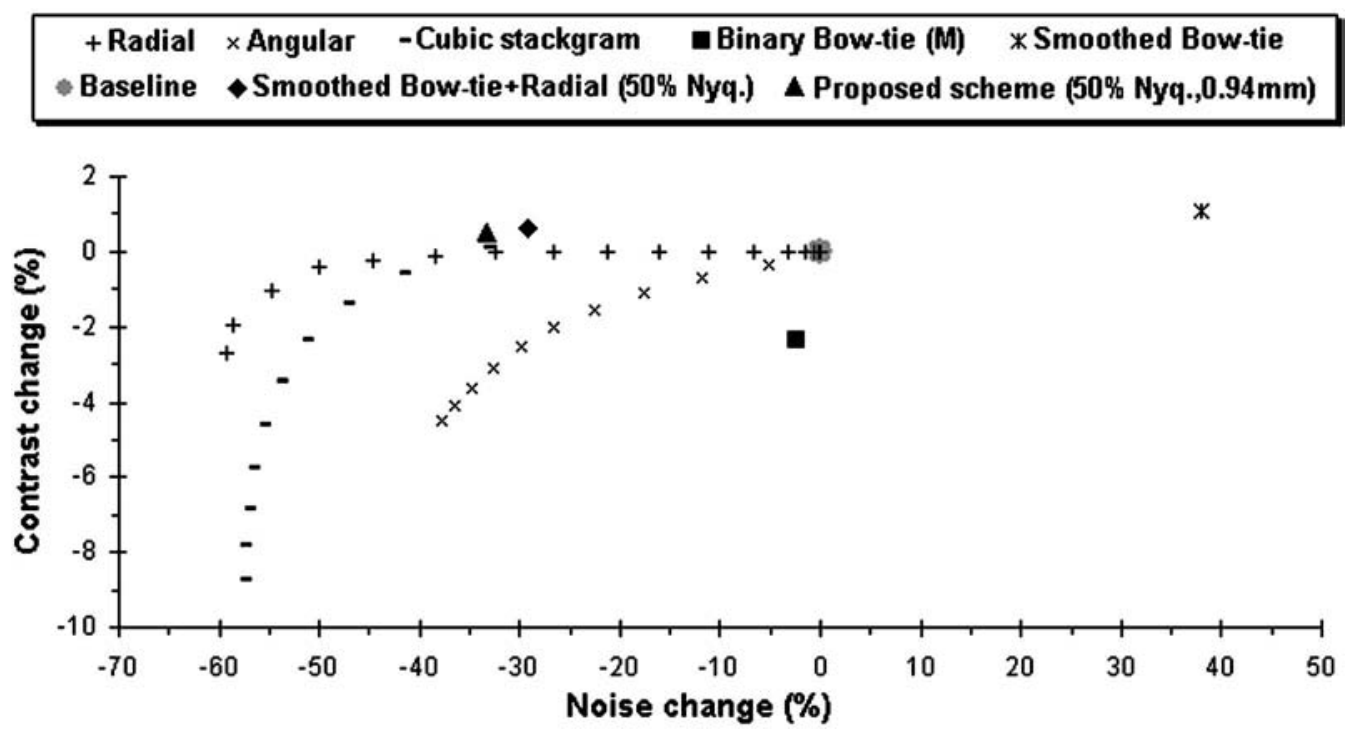

FIG. 5. Results in contrast vs noise for the filters in Table I.

degradation $(3 \%)$ as well as a loss of tangential resolution (46\% at $10 \mathrm{~mm}$ from the center of the FOV).

When using the smallest size of the Gaussian kernel in the stackgram domain (depicted as "-" in Figs. 5 and 6), we achieved a noise reduction of $33 \%$ at the cost of degrading radial and tangential resolutions $(14.5 \%$ and $16 \%$, respectively).

The use of a binary bow-tie mask [ $M$ defined in Eq. (1), depicted as " $\square$ " in Figs. 5 and 6] reduced noise by 3\% although contrast decreased and no noticeable gain in resolution was obtained as compared with the baseline reconstruction. The smoothed bow-tie filter (bow-tie mask smoothed with a ten-point Gaussian kernel, depicted as " *" in Figs. 5 and 6) showed a gain in both radial and tangential resolution (4\% and $17 \%$, respectively) and contrast $(1 \%)$, at the cost of an increase in noise of $38 \%$. This increase in noise can be compensated by the addition of a low-pass radial filter (depicted as " $"$ in Figs. 5 and 6). Moreover, to achieve a more isotropic result, our proposed filtering scheme also incorporates an axial filter (depicted as " $\boldsymbol{\Delta}$ " in Figs. 5 and 6). Axial filter (FWHM=0.94 mm) reduced noise by $5 \%$ at the cost of degrading axial resolution by $6 \%$, while radial and tangential resolution and contrast remained unaffected. Since all the methods studied are intrinsically 2D, combination with an axial filter is a good option to obtain a more isotropic result.

For a noise reduction of $33 \%$, our bow-tie filtering scheme (depicted as " $\boldsymbol{\Delta}$ " in Figs. 5 and 6) showed better results than any of the other filters studied in terms of both resolution (improvement of $10 \%$ in radial resolution with no noticeable change in tangential resolution) and contrast (1\% increase).

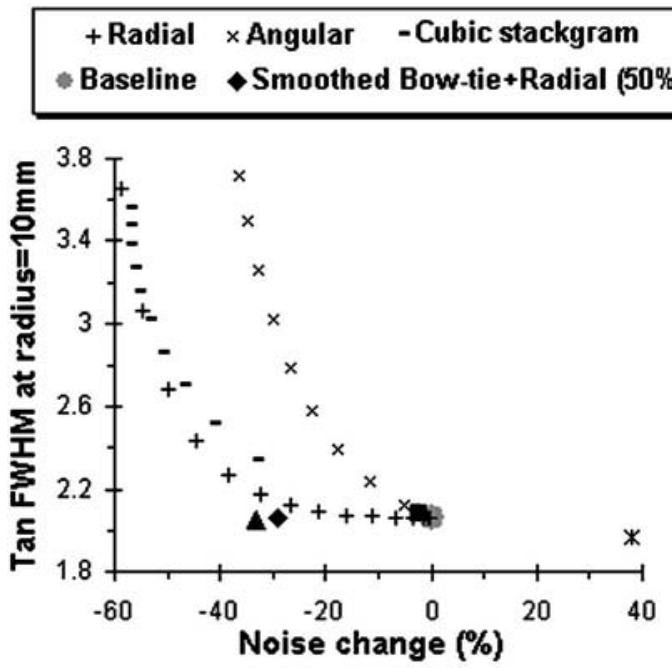

-Binary Bow-tie (M) $\quad *$ Smoothed Bow-tie

A Proposed scheme (50\% Nyq.,0.94mm)

Table I. 


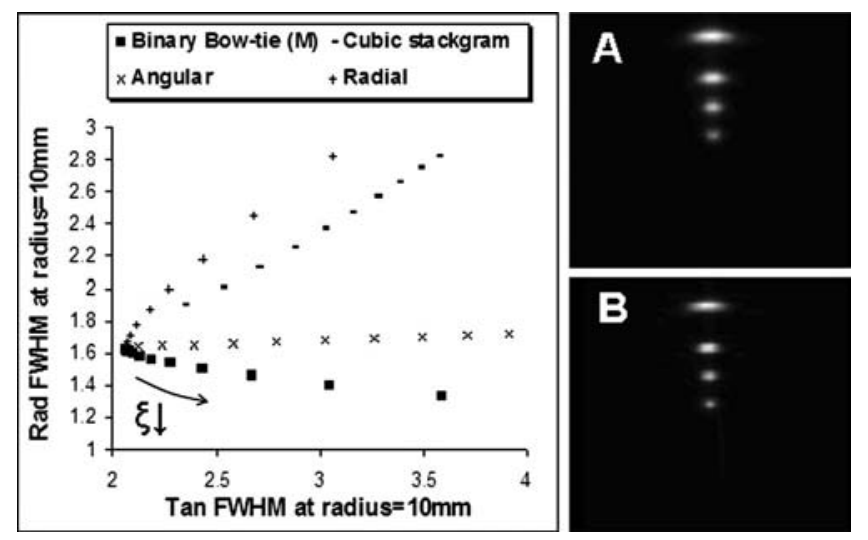

FIG. 7. Left: Results in radial vs tangential resolution, measured as FWHM of a point placed $10 \mathrm{~mm}$ from the center of the FOV for radial, angular, stackgram, and binary bow-tie filters. Right: (a) Four point sources filtered with a Gaussian angular filter FWHM $=10^{\circ}$. (b) Four point sources filtered with a bow-tie filter for a $\xi$ value of 0.4 (too low for the points far from the center). The same position-dependent blurring can be observed in both cases.

Regarding isotropy, it can be seen from Fig. 7, left, that the angular filter (" $X$ " in the plot) is highly anisotropic, producing, at positions far from the center of the FOV $(10 \mathrm{~mm}$ in the plots), a higher resolution degradation in the tangential direction than in the radial direction. The same effect can be seen when the bow-tie mask is too small since $\xi$ controls the cutoff frequency in the angular direction ("ם" in the plot).
This anisotropic behavior resulted in a noticeable artifact appearing as circular blurring dependent on the distance to the center of the FOV both for angular filter [Fig. 7(a), right], and for the bow-tie filter with low values of $\xi$ [Fig. 7(b), right].

Experiments performed with rodent brain studies allowed us to verify the enhancement in image quality achieved when using our filtering scheme (Figs. 8 and 9). The parameters for each filter in this comparison were visually selected by an experienced user.

The proposed filtering scheme also enhanced the resolution of small structures, such as a mouse heart (Fig. 10). The baseline reconstruction included a radial Butterworth filter, with order of 12 and cutoff frequency of $45 \%$ Nyquist, combined with an axial filter. Figure 11 shows the profiles corresponding to the dashed lines in Fig. 10 normalized to their maximum value. The peak-to-valley ratio measured on a profile across the myocardium increased from 1.1 to 2.1 after adding the bow-tie filter with $\xi=0.30$ to the baseline reconstruction.

\section{DISCUSSION AND CONCLUSIONS}

This paper presents the results of a new filtering strategy, based on the expected shape of the sinogram in the Fourier transform domain of sinogram data. Our "bow-tie filter scheme" comprises a first filtering stage based on a binary bow-tie mask smoothed with a Gaussian kernel, followed by

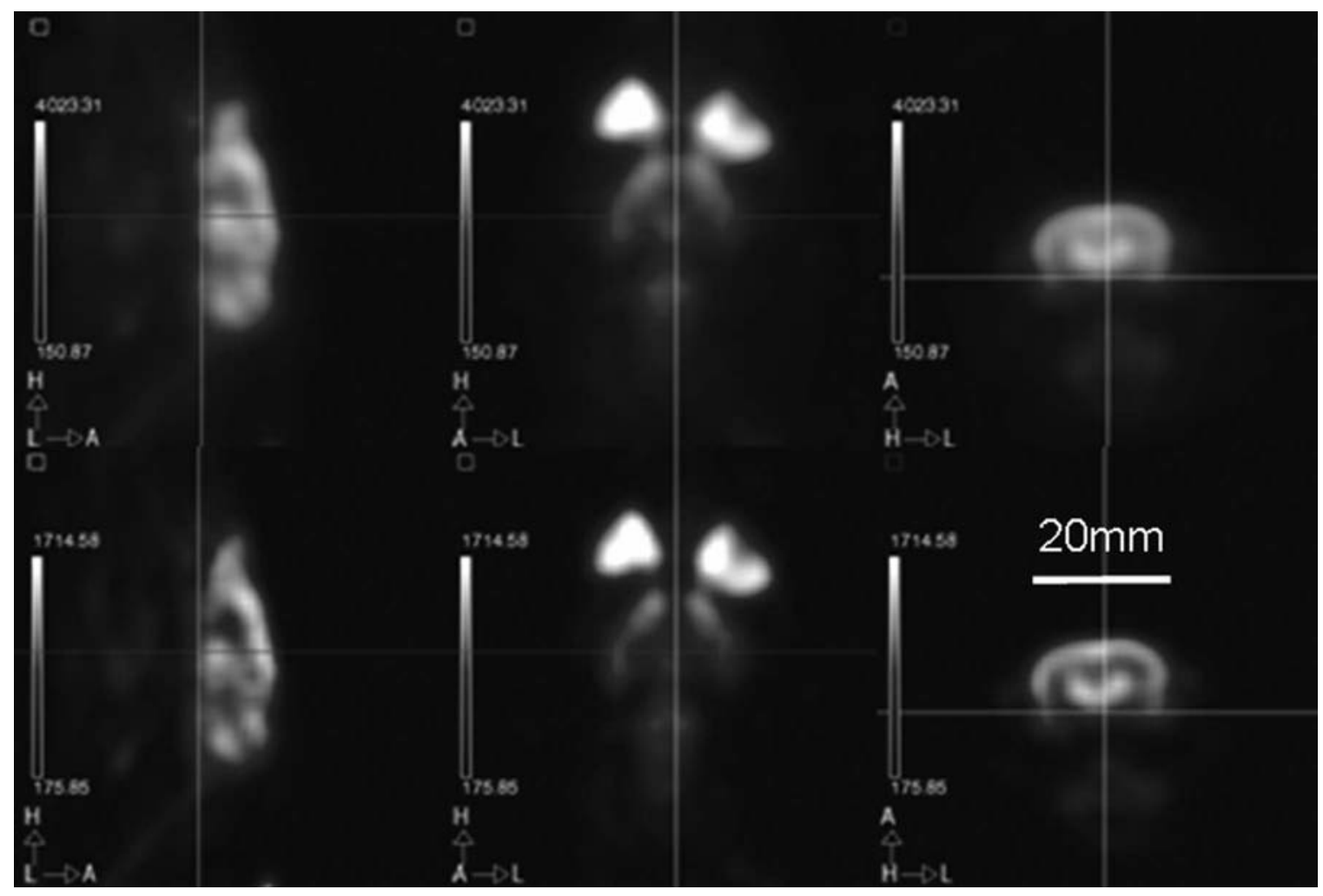

FIG. 8. Coronal, sagittal, and axial views of an FDG rat brain study. Upper panel: Radial filter (Butterworth, 12th-order, cutoff frequency $35 \%$ Nyquist) + axial filter (Gaussian, FWHM $0.94 \mathrm{~mm}$ ). Lower panel: Proposed filtering scheme: Bow-tie filter $(\xi=0.50)+$ radial filter (Butterworth, 12th-order, cutoff frequency $35 \%$ Nyquist) + axial filter (Gaussian, FWHM $0.94 \mathrm{~mm}$ ). 


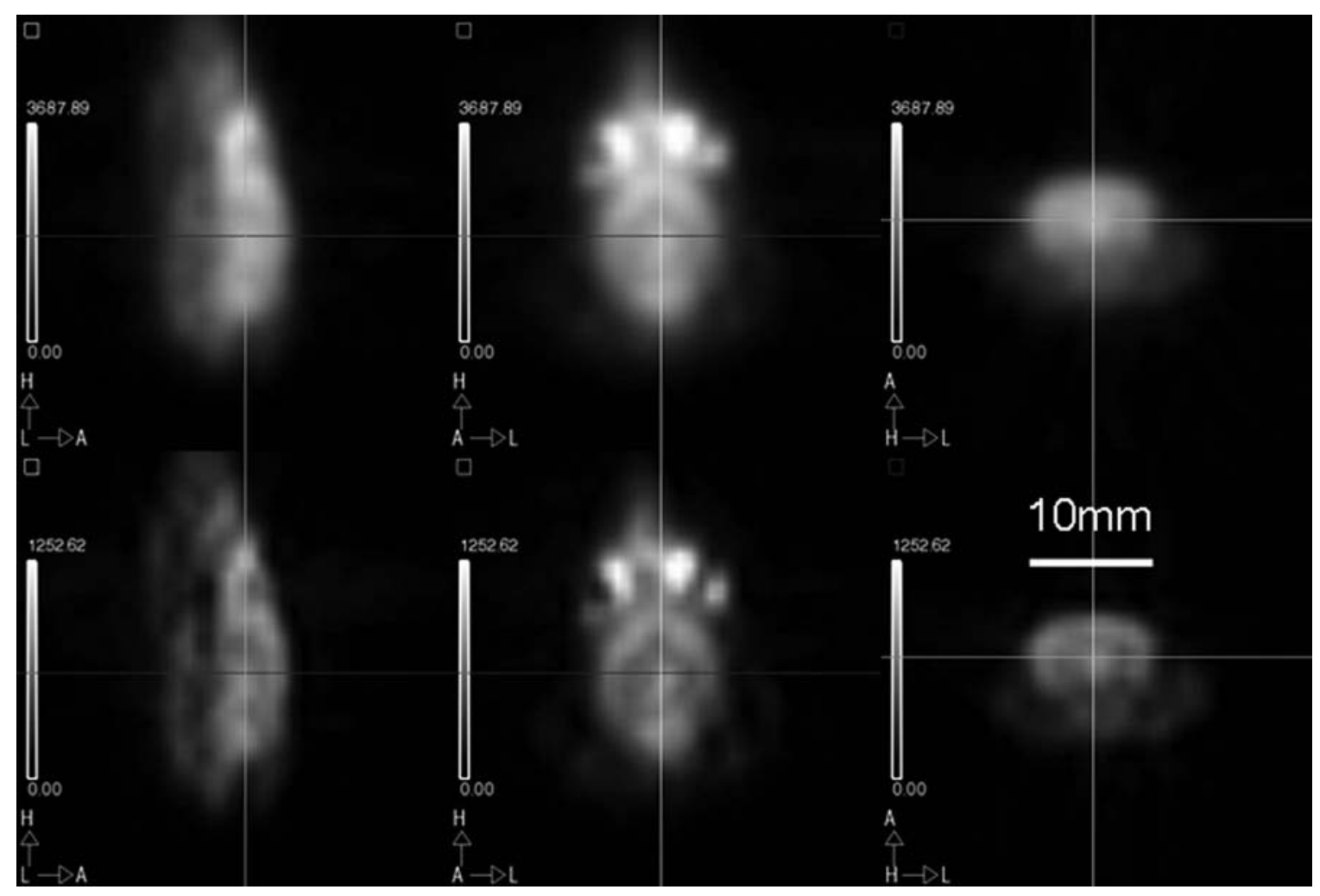

FIG. 9. Coronal, sagittal, and axial views of an FDG mouse brain study. Upper panel: Radial filter (Butterworth, 12th-order, cutoff frequency 35\% Nyquist) + axial filter (Gaussian, FWHM $0.94 \mathrm{~mm}$ ). Lower panel: Proposed filtering scheme: Bow-tie filter $(\xi=0.35)+$ radial filter (Butterworth, 12th-order, cutoff frequency 35\% Nyquist) + axial filter (Gaussian, FWHM $0.94 \mathrm{~mm}$ ).

a conventional Butterworth radial filter and a Gaussian filter in the axial direction. The performance of this filtering scheme over a baseline reconstruction (ramp filter only) is compared with that provided by other filtering strategies proposed in literature: radial, angular, axial, and stackgramdomain filtering.

As is well known, smoothing in the radial direction reduces noise, which is increased at high frequencies by the ramp-filtering step in the FBP reconstruction. As an intense radial filtering introduces considerable blurring in the image, a more efficient filtering strategy is clearly desirable.

In our study, the use of isolated angular, radial, and stackgram filters reduced noise but at the cost of a severe loss of resolution (Fig. 6) and reduced contrast (Fig. 5). The loss of resolution was similar for radial and tangential components when applying radial and stackgram filters, but not in the case of applying an angular filter (Fig. 7, left), which resulted in noticeable circular blurring, that was dependent on the distance to the center of the FOV (Fig. 7, right), as reported elsewhere. ${ }^{20,1,2}$ Several approaches have been proposed to overcome this problem. Stearns suggested a metric to enable an automatic determination of the optimal parameters for angular filtering, based on the information about object shape and the amount of radial filtering applied. ${ }^{20}$ Andía et $a .^{21}$ proposed a scheme with variable-length linear angular filters dependent on the distance from the center of the FOV.

For stackgram filtering, there was an important low-pass effect (degradation of resolution and noise reduction), before the application of any filter, due to the interpolations involved in the transformation to and from the stackgram domain (Fig. 4). We tested three different interpolation schemes, and cubic interpolation showed the best performance, in agreement with other authors. ${ }^{22}$ Another drawback of stackgram-domain filtering is its computational burden, which is five times higher than that of any of the other methods for cubic interpolation. These drawbacks of stackgram filtering have been addressed in a new approach presented by Peltonen and Ruotsalainen, ${ }^{23}$ who proposed a new sinogramdomain filtering which approximates the stackgram filter, thus eliminating the need for transformations between the sinogram and stackgram domains.

The noise reduction of $4 \%$ achieved by the constantamplitude binary bow-tie mask is probably due to the removal of inconsistent events from the sinogram (those located outside the expected area in the Fourier domain). Further noise reduction was achieved in our filtering scheme by including radial and axial filters (Figs. 5 and 6). The advantage of including an axial filter is that it provides noise reduction without degrading transaxial resolution, thus improving isotropy.

The bow-tie filtering scheme proposed in this paper yielded excellent results in terms of resolution, contrast, and peak-to valley ratio. The increase in resolution probably comes from the pre-emphasis component of the filter, which slightly reduces the low frequencies. We believe that this pre-emphasis could also decrease the influence of scatter in 


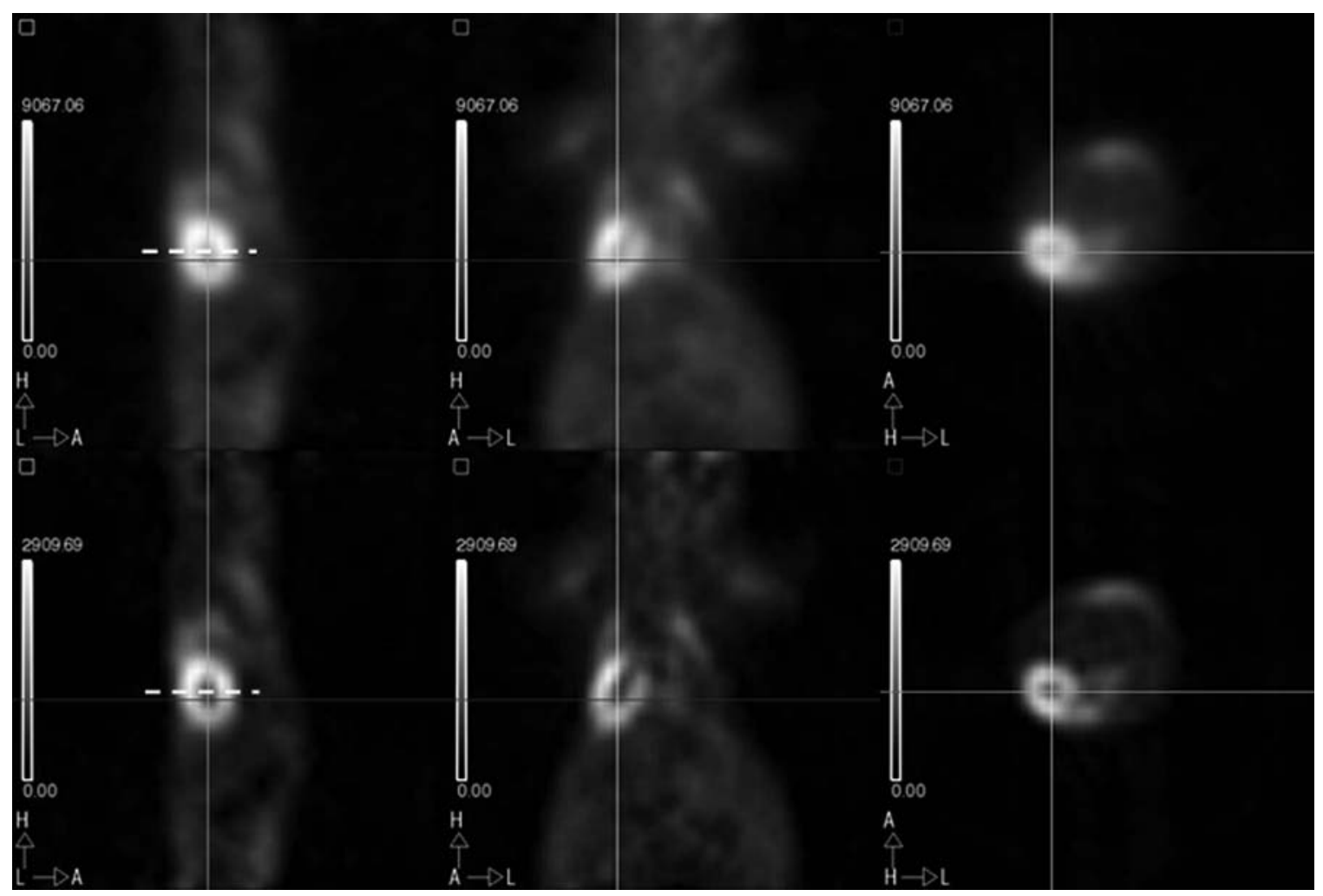

FIG. 10. Coronal, sagittal, and axial views of an FDG mouse heart study. Upper panel: Radial filter (Butterworth, 12th-order, cutoff frequency 45\% Nyquist) + axial filter (Gaussian, FWHM $0.94 \mathrm{~mm}$ ). Lower panel: Proposed filtering scheme: Bow-tie filter $(\xi=0.30)+$ radial filter (Butterworth, 12th-order, cutoff frequency $45 \%$ Nyquist) + axial filter (Gaussian, FWHM $0.94 \mathrm{~mm}$ ).

the image, thus helping to reduce image glare. The rationale is that, since scatter can typically be modeled by a wide Gaussian, ${ }^{24,25}$ its Fourier transform would correspond to a peak in the low frequency range. This could also explain the enhancement observed in contrast and peak-to-valley ratio.

Parameter $\xi$ in the smoothed bow-tie filter should be adjusted to the size of the object for optimum behavior. If it is too small, the filtering scheme produces an effect similar to that of using excessive angular filtering since $\xi$ controls the cutoff frequency in the angular direction. An easy automatic

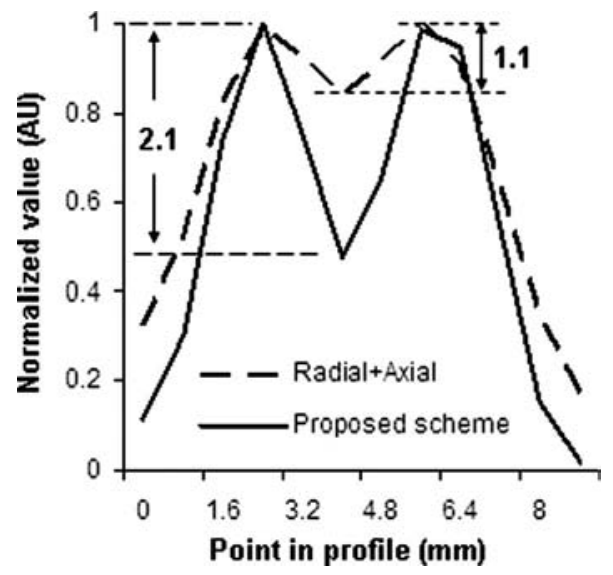

FIG. 11. Normalized profiles corresponding to the dotted lines in Fig. 10: Dashed line, radial+axial filtering; solid line, radial+bow-tie+axial filter (proposed method). Arrows indicate the peak-to-valley ratio. procedure could be implemented to obtain the optimum value for this parameter by extracting the boundaries of the image from two orthogonal projections.

We tested a wide range of values for the other parameters of our proposed filtering scheme, i.e., type and cutoff frequency (radial and axial filters). We present only one set of results for the sake of clarity in the plots, although these were selected visually by an experienced user, as is commonly the case in practice.

The promising results with the bow-tie filtering scheme presented in this paper were obtained with a very straightforward implementation of the filter: A windowed binary mask with smoothed edges and pre-emphasis. Presumably, better results could be obtained with a more refined implementation, optimized to better remove scatter and randoms components. Another interesting issue that warrants further study is the effect of this filtering scheme on the quantitative properties of the resulting image.

In summary, we assessed the performance of what we call a bow-tie filtering scheme, which comprises a bow-tie mask smoothed with a Gaussian kernel in combination with a conventional Butterworth radial filter and a Gaussian axial filter. A thorough comparison is presented against other filtering strategies proposed in nuclear medicine. The bow-tie filtering scheme was the best option, achieving the necessary noise reduction with the best contrast and reducing degradation of resolution. Experiments on rodent images showed a noticeable improvement in visual image quality. 


\section{ACKNOWLEDGMENTS}

This work was partially funded by projects CD-TEAM (CENIT program, Ministerio de Industria), CIBERsam CB07/09/0031 (Ministerio de Sanidad y Consumo), TEC2004-07052-C02 (Ministerio de Educación y Ciencia), and GR/SAL/024104 (Comunidad de Madrid).

a) URL: www.hggm.es/image.

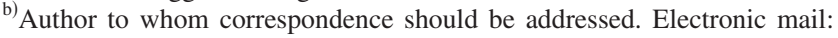
desco@mce.hggm.es; Also at Laboratorio de Imagen, Unidad de Medicina Experimental, Hospital General Universitario Gregorio Marañón, C/Doctor Esquerdo, 46, 28007 Madrid, Spain. Telephone: + 34915866678; Fax: +34914265108.

${ }^{1}$ M. E. Daube-Witherspoon and R. E. Carson, "Investigation of angular and axial smoothing of PET data," IEEE Nuclear Science Symposium Conference Record, 1996, Vol. 3, pp. 1557-1561.

${ }^{2}$ M. E. Daube-Witherspoon and R. E. Carson, "Investigation of angular smoothing of PET data," IEEE Trans. Nucl. Sci. 44, 2494-2499 (1997).

${ }^{3}$ A. Chatziioannou and M. Dahlbom, "Detailed investigation of transmission emission data smoothing protocols and their effects on emission images," IEEE Trans. Nucl. Sci. 43, 290-294 (1996).

${ }^{4}$ A. P. Happonen and S. Alenius, "Sinogram filtering using a stackgram domain," Proceedings of the Second IASTED International Conference: Visualization, Imaging and Image Processing, 2002, pp. 339-343.

${ }^{5}$ A. P. Happonen and S. Alenius, "A comparison of sinogram and stackgram domain filtering methods employing L-filters for noise reduction of tomographic data," Proceedings of the 2005 Finnish Signal Processing Symposium, 2005, pp. 1-4.

${ }^{6}$ P. A. Rattey and A. G. Lindgren, "Sampling the 2-D Radon transform," IEEE Trans. Acoust., Speech, Signal Process. ASSP-29, 994-1002 (1981).

${ }^{7}$ P. R. Edholm, R. M. Lewitt, and B. Lindholm, "Novel properties of the Fourier decomposition of the sonogram," Workshop on Physics and Engineering of Computerized Multidimensional Imaging and Processing, 1986, Vol. 671, pp. 8-18.

${ }^{8}$ M. Defrise, "A factorization method for the 3D X-ray transform," Inverse Probl. 11, 983-994 (1995).

${ }^{9}$ M. Defrise, P. E. Kinahan, and D. W. Townsend, "Exact and approximate rebinning algorithms for 3DPET data," IEEE Trans. Med. Imaging 16, 145-158 (1997).

${ }^{10} \mathrm{~F}$. Natterer, The Mathematics of Computerized Tomography (Wiley, New York, 1986).

${ }^{11}$ J. S. Karp, G. Muehllehner, and R. M. Lewitt, "Constrained Fourier space method for compensation of missing data in emission computed tomog- raphy," IEEE Trans. Med. Imaging 7, 21-25 (1988).

${ }^{12}$ W. Xia, R. M. Lewitt, and P. R. Edholm, "Fourier correction for spatially variant collimator blurring in SPECT," IEEE Trans. Med. Imaging 14, 100-115 (1995)

${ }^{13}$ B. I. Andía, "Nonstationary and nonlinear sinogram filtering for tomographic image reconstruction," Ph.D. thesis, Department of Electrical Engineering, Notre Dame University, Indiana, 2003.

${ }^{14}$ J. J. Vaquero, A. Molins, J. Ortuño, J. Pascau, and M. Desco, "Preliminary results of the small animal rotational positron emission tomography scanner," Mol. Imaging Biol. 6, 102 (2004).

${ }^{15}$ J. J. Vaquero, E. Lage, L. Ricón, M. Abella, E. Vicente, and M. Desco, "rPET detectors design and data processing," IEEE Nuclear Science Symposium Conference Record, 2005, Vol. 5, pp. 2885-2889.

${ }^{16}$ M. E. Daube-Witherspoon and G. Muehllehner, "Treatment of axial data in three dimensional PET," J. Nucl. Med. 28, 1717-1724 (1987).

${ }^{17}$ A. P. van der Weerdt, R. Boellaard, P. Knaapen, C. A. Visser, A. A. Lammertsma, and F. C. Visser, "Postinjection transmission scanning in myocardial 18F-FDG PET studies using both filtered backprojection and iterative reconstruction," J. Nucl. Med. 45, 169-175 (2004).

${ }^{18}$ K. Lee, P. E. Kinahan, J. A. Fessler, R. S. Miyaoka, M. Janes, and T. K. Lewellen, "Pragmatic fully 3D image reconstruction for the MiCES mouse imaging PET scanner," Phys. Med. Biol. 49, 4563-4578 (2004).

${ }^{19}$ S. España, J. L. Herraiz, E. Vicente, E. Herranz, J. J. Vaquero, M. Desco, and J. M. Udias, "Improved image reconstruction in small animal PET using a priori estimates of single-pixel events," Proceedings of the IEEE Nuclear Science Symposium Conference Record, 2007, Vol. 5, pp. 38763880 .

${ }^{20} \mathrm{C}$. W. Stearns, "Context-sensitive angular filtering of PET transmission data," Proceedings of the IEEE Medical Imaging Conference Record, 1995, Vol. 13, pp. 1332-1334.

${ }^{21}$ B. I. Andía, K. D. Sauer, and C. A. Bouman, "Radially-dependent nonlinear sinogram filtering," Proceedings of the IEEE Nuclear Science Symposium Conference Record, 2001, Vol. 3, pp. 1404-1408.

${ }^{22}$ T. M. Lehmann, C. Gonner, and K. Spitzer, "Survey: Interpolation methods in medical image processing," IEEE Trans. Med. Imaging 18, 10491075 (1999).

${ }^{23}$ S. Peltonen and U. Ruotsalainen, "New sinogram filter design utilizing sinusoidal trajectories," Proceedings of the IEEE Nuclear Science Symposium Conference Record, 2006, Vol. 5, pp. 2770-2774.

${ }^{24}$ Y. Yang and S. R. Cherry, "Observations regarding scatter fraction and NEC measurements for small animal PET," IEEE Trans. Nucl. Sci. 53, 127-132 (2006).

${ }^{25}$ H. Zaidi and K. F. Koral, "Scatter correction strategies in emission tomography," Quantitative Analysis in Nuclear Medicine Imaging (Springer, New York, 2006), pp. 205-235. 